\title{
Developmental changes in the responsiveness to ecdysterone of chromosome region I-18C of Chironomus tentans
}

\author{
Markus Lezzi ${ }^{1}$, Friederike Gatzka ${ }^{1}$, and Michel Robert-Nicoud ${ }^{2}$ \\ ${ }^{1}$ Institute for Cell Biology, Swiss Federal Institute of Technology, Hönggerberg, CH-8093 Zürich, Switzerland \\ ${ }^{2}$ Department of Molecular Biology, Max Planck Institute for Biophysical Chemistry, P.O. Box 2841, D-3400 Göttingen, \\ Federal Republic of Germany
}

\begin{abstract}
The presence of nascent RNA in chromosome region $\mathrm{I}-18 \mathrm{C}$ was revealed by the method of induced hybrid formation (IEH) and by use of anti-RNA:DNA antibodies. IEH signals were quantitated, and used as a measure of transcriptional activity in the region. The expansion of the I-18C region was also determined and used as an indication of local chromatin decondensation. Chironomus tentans larvae may undergo oligopause or they may develop subitaneously. It was found that the course of basal activity in I-18C differs substantially in these two modes of development. Most interestingly they also differ in activation of $\mathrm{I}-18 \mathrm{C}$ in response to in vitro ecdysterone treatment. Changes in hormonal responsiveness of I-18C parallel changes in the preexisting state of I-18C decondensation. Oligopause stage 5 animals, being most refractory in this respect, exhibit the most condensed I-18C chromosome region.
\end{abstract}

\section{Introduction}

Chromosome region $\mathrm{I}-18 \mathrm{C}$ of Chironomus (Camptochironomus) tentans is the first region in which experimentally a puff could be induced by ecdysone, i.e., by injection of the purified hormone into intermolt larvae (Clever and Karlson 1960). Subsequent work by Clever et al. (1973) has shown that this puff induction also occurs in vitro. When we set out to reproduce these experiments we encountered the problem that puff $\mathrm{I}-18 \mathrm{C}$ could be induced in some cases and not in others. In order to explain this variability we first learned to distinguish between the two aspects of puffing, i.e., activation of transcription and local chromatin decondensation. Second, we learned to observe and to control various parameters which obviously influence $\mathrm{I}-18 \mathrm{C}$ activation in response to hormone application (a preliminary note is given in Lezzi and Richards 1989). In the present full report we demonstrate that the mode and the precise stage of development of fourth instar larvae of $C$. tentans are such parameters. In subsequent papers of this series other parameters will be discussed. The aim of these comprehensive studies is the elucidation of the hormone dependent gene activation process in its whole complexity and regulatory fine tuning.

\section{Materials and methods}

\section{Animals}

Strain $2 \mathrm{~L}_{\mathbf{k}}$ of $C$. tentans was used for the present work. It carries a male-specific complex inversion in the left arm of chromosome 2 which enables the sexes to be distinguished on the basis of chromosomal squashes (cf. Beermann 1955). No significant sex-specific differences were observed in the present studies on chromosome region I-18C. The larvae were raised as described by Meyer et al. (1983). The daily light period for long day conditions lasted from 4 a.m. to 10 p.m. (Central European Time), and that for short day conditions from 7 a.m. to 1 p.m. The larvae used were sacrificed between $11 \mathrm{a} . \mathrm{m}$. and $12.30 \mathrm{a} . \mathrm{m}$. They were staged as described by Ineichen et al. (1983) carefully avoiding long lasting changes in their temperature, illumination and living conditions. Oligopausing animals were selected on the basis not only of life history and rate of development but also of their reduced motile activity.

\section{Antisera}

The goat antibodies against RNA:DNA hybrids have been characterized previously (Kitagawa and Stollar 1982; Büsen et al. 1982; Alcover et al. 1982). The bona fide "anti-RNP antiserum" is a rabbit serum originally raised against ecdysteroids (see Dworniczak et al. 1983) and which we found to exhibit the following additional characteristics. It reacted with puffs and Balbiani rings of fixed or unfixed chromosomes. The reactivity of these chromosomal sites was lost when unfixed chromosomes (Widmer et al. 1984) were digested with RNase A $(0.1 \mathrm{mg} / \mathrm{ml})$ in Glancy medium ( $90 \mathrm{mM} \mathrm{KCl}, 40 \mathrm{mM} \mathrm{NaCl}, 20 \mathrm{mM}$ triethanolamine, $\mathrm{pH} 7.6$ ) for $30 \mathrm{~min}$ at room temperature. However, RNase treatment of fixed chromosomes did not result in such a loss (the success of RNase digestion was verified autoradiographically after $\left[{ }^{3} \mathrm{H}\right]$ uridine incorporation). We interpret these findings as resulting from the presence of epitopes in structures which are linked to the native chromosome via (nascent) RNA molecules, and which become bonded to chromosomes in an RNase-resistant form as a result of formaldehyde fixation. For all these reasons we deemed it justified to use the anti-ecdysteroid antiserum as an indicator for RNP structures and to call it anti-RNP antiserum in the present work. Rhodamine-labeled rabbit anti-goat and fluorescein isothiocyanate (FITC) labeled goat antirabbit sera were from Cappel (Pa, USA).

\section{Injection of larvae}

Larvae were narcotized in a 1:200 solution of MS 222 (Sandoz). Injection was accomplished essentially as described by Lezzi and Kroeger (1966). Control larvae received $1 \mu 1$ of $0.65 \% \mathrm{NaCl}$ solution, and experimental larvae, $1 \mu \mathrm{g} / \mathrm{ml}$ 
ecdysterone (Rohto, Japan) in the same solution. Injected larvae were placed individually in beakers containing substrate and water from their previous cultivation pan. The beakers were aerated and kept at the temperature of their original cultivation pan for $24 \mathrm{~h}$. Our analysis included only those larvae which were completely buried in the substrate.

\section{In vitro cultivation of salivary glands}

Pairs of larval salivary glands were dissected and incubated as described by Lezzi (1984). Incubation was at $18^{\circ} \mathrm{C}$ for $1 \mathrm{~h}$ in $83 \%$ ZO-medium (Wyss 1982) containing $1.6 \times 10^{-5} \mathrm{M}$ ecdysterone and $1.5 \%$ ethanol. Pulse labeling with $\left[{ }^{3} \mathrm{H}\right]$ uridine was done as described by Lezzi et al. (1984).

\section{Cytological, autoradiographical and immunohistochemical preparations}

The preparation of orcein/fast green-stained squashes has been described by Lezzi et al. (1981). Autoradiography was in principle carried out as described by Lezzi et al. (1984) except that the chromosomal squashes were made on $24 \times 32 \mathrm{~mm}$ coverslips (instead of slides) which were stained after the photographic fixation step with $0.01 \%$ berberine sulfate (Fluka, Switzerland) in 1/10 Mcllvaine buffer, pH 3.8. The coverslips were mounted in Entellan (Merck) with the chromosomes facing the microscopic slide. This inverted arrangement allowed the chromosomes to be viewed by epifluorescence illumination (FITC filter setting, $40 \times$ Neofluar objective, Zeiss) without interference by overlaying silver grains.

For detection of induced endogenous hybrids (IEH) between nascent RNA and chromosomal DNA the chromosomes were prepared and processed essentially according to Alcover et al. (1982) with the modifications described by Hertner et al. (1986). Salivary glands were rinsed and incubated in NKE $(100 \mathrm{mM} \mathrm{NaCl}, 5 \mathrm{mM} \mathrm{KCl}, 0.5 \mathrm{mM}$ EDTA, $10 \mathrm{mM}$ Tris-maleate, $\mathrm{pH}$ 6.3) in order to solubilize the secretion from the opened lumen (Hertner et al. 1980). They were then incubated for $1 \mathrm{~min}$ in ice-cold Glancy medium containing $0.1 \%$ Triton $X-100$, transferred into $50 \%$ acetic acid, and squashed. The siliconized coverslip was pricked off after freezing on dry ice, and the slide was immediately immersed in 100\% ethanol (two changes) and airdried. The preparations were heat-fixed in $2 \times$ SSC $(1 \times$ SSC is $150 \mathrm{mM} \mathrm{NaCl}, 15 \mathrm{mM}$ sodium citrate) for $30 \mathrm{~min}$ at $58^{\circ} \mathrm{C}$, and rinsed in two changes each of $96 \%$ and $100 \%$ ethanol before air-drying. They were then denatured at $85^{\circ} \mathrm{C}$ for $30 \mathrm{~s}$ and renatured in hybridization solution (see Method 2 of Langer-Safer et al. 1982) at $58^{\circ} \mathrm{C}$ for $10 \mathrm{~min}$. Before and after the reannealing step the slides were rinsed in $5 \times \mathrm{SSC}$ at $85^{\circ} \mathrm{C}$ and $58^{\circ} \mathrm{C}$, respectively; subsequently they were washed in $2 \times \mathrm{SSC}$ (three times $20 \mathrm{~min}$ ) at $53^{\circ} \mathrm{C}$, and twice for $2.5 \mathrm{~min}$ in PBS $(0.01 \mathrm{mM}$ phosphate buffer, $0.15 \mathrm{M} \mathrm{NaCl}, \mathrm{pH}$ 7.5) at room temperature. The chromosomal squashes were covered with $15 \mu$ of PBS containing $10 \mu \mathrm{g} / \mathrm{ml} \mathrm{BSA}$ (bovine serum albumin) under a $24 \times 32 \mathrm{~mm}$ coverslip, and subsequently kept in a moist chamber at room temperature before they were incubated with antihybrid antiserum ( $50 \times$ diluted) for $1 \mathrm{~h}$ under the same conditions. Before and after incubation with the rhodaminelabeled antiserum $(100 \times$ diluted $)$ under the same conditions, the slides were washed four times with PBS for $4 \mathrm{~min}$ each. The preparations were mounted in glycerol, sealed with nail polish and stored at $-20^{\circ} \mathrm{C}$ for up to 3 years.

For immunohistochemical studies with the anti-RNP serum the chromosomes were either fixed and prepared according to Dworniczak et al. (1983) or they were isolated in an unfixed state according to Widmer et al. (1984). The immunological procedure has been described by Dworniczak et al. (1983).

\section{Evaluation of chromosome region $\mathrm{I}-18 \mathrm{C}$}

Orcein/fast green-stained preparations. Since local decondensation of Chironomus salivary gland chromosomes results primarily in longitudinal rather than lateral expansion of the respective region, the relative length $\left(\mathrm{L}_{\mathrm{r}}\right)$ of region I- $18 \mathrm{C}$ could be taken as a measure of its state of condensation. This was determined as described by Lezzi (1974). Only unstretched chromosomes I were evaluated which exhibited a ratio of total length to average width of less than 12 and which exhibited no bent or oblique bands and no hourglass-shaped interband regions with longitudinal striation.

Autoradiograms. Grains were counted over chromosome region $\mathrm{I}-18 \mathrm{C}$ and reference region $\mathrm{r}$ (for exact delineation of these regions see Lezzi 1974) in transmitted light (objective lens $40 \times$ ). Since the labeled area of the adjacent puff in $\mathrm{I}-19 \mathrm{~A}_{1}$ was often not separated from that of puff I-18C it was important to be able to visualize the banding pattern of chromosomes as clearly as possible. This could be accomplished by simultaneous or alternating epillumination of the (inverted) autoradiogram stained with fluorescent dye (see Fig. 1). Grain numbers from 0 to 10 per I-18C region were usual and ideal for counting. Grain numbers from 11 to 30 were given lower weights (factors of $1 \times$ to $0.3 \times$, respectively); grain numbers in $\mathbf{r}$ were corrected accordingly. Grain numbers above 30 were not included in the analy-
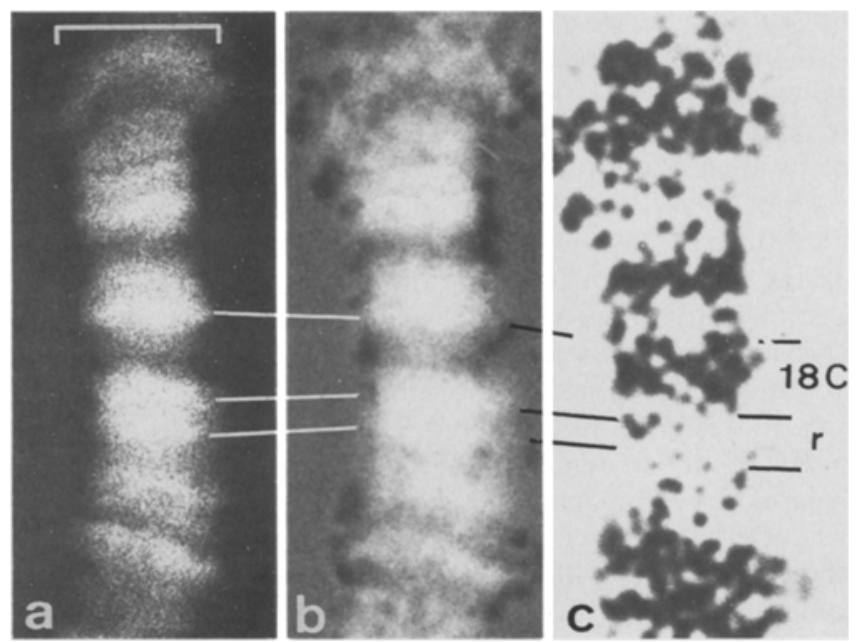

Fig. 1 a-c. Inverted autoradiogram of the right tip of chromosome I after $\left[{ }^{3} \mathrm{H}\right]$ uridine incorporation in the presence of ecdysterone. Animal was in first half of stage 9. Chromosome stained with fluorescent dye. a Epifluorescence illumination. $\mathbf{b}$ Combined epifluorescence and transmitted illumination. c Transmitted illumination. Region $\mathrm{I}-18 \mathrm{C}$ and reference region $(r)$ are indicated. Note that grain density in $\mathrm{I}-18 \mathrm{C}$ is at the upper limit of that tolerated for grain counting (for details see Materials and methods). Bar represents $10 \mu \mathrm{m}$ 
sis because grains tended to form aggregates. The sum of the corrected grain numbers of all evaluated $\mathrm{I}-18 \mathrm{C}$ regions per animal was divided by the sum of the corrected grain numbers of the respective reference regions to yield $G_{r}$. This cumulative procedure was necessary because quite often the individual chromosome exhibited no grains in I-18C or in $r$. Obviously this procedure precludes the possibility of statistical analysis. $\mathrm{G}_{\mathrm{r}}$-values of animals belonging to the same experimental group were averaged.

\section{Immunohistochemical preparations}

For visual scoring, chromosomes I were photographed on Ilford HP5 film with epifluorescence illumination (setting for the respective fluorescent dye) using, as a rule, a PlanNeofluar $63 \times$ objective with built-in diaphragm (Zeiss) and an ocular magnification of $12.5 \times$. Phase contrast photographs were taken after each exposure without changing magnification. About 10 chromosomes were photographed per slide. Photographic prints were made using grade 4 paper (Ilfoprint or Tetanal) at a size of $9 \times 13 \mathrm{~cm}$. The I-18C region was localized with the aid of the phase contrast photograph and marked with ink. Photographs of one experimental series were rendered anonymous, mixed and scored by comparison with reference photographs. Figure 2 illustrates the most typical appearance of each of the I-18C signal classes between $0-19$. Since the signal pattern and the general signal as well as background intensities varied considerably between different series we routinely used for comparison four, instead of just one, reference pictures per class, each exhibiting larger portions of the chromosomal complement than those shown in Figure 2. The intensity and area of fluorescent signal in I-18C were judged in relation to those of neighboring chromosome regions which in turn were compared with more distant landmarks. The scores of all chromosomes evaluated from all animals from one stage and/or treatment were averaged to yield the relative fluorescence value $\left(F_{r}\right)$ for $\mathrm{I}-18 \mathrm{C}$ and the $95 \%$ confidence limits.

Computer-assisted image analyses were performed at the Department of Molecular Biology of the Max Planck Institute for Biophysical Chemistry in Göttingen. The same equipment was used as described by Arndt-Jovin et al. (1985; "Quantitative fluorescence image analysis system" section of Materials and Methods). In short, epifluorescent images were collected by use of a microscope equipped with a Plan-Neofluar $63 \times$ objective (Zeiss) and a Cohu (San Diego, Calif) series 4410 double-intensified Newicon TV camera. The signals were transferred to an LSI/11 computer and analyzed using the Incos software system (Signum, Munich). The images were acquired and processed as follows: averaging over 256 frames; correction for unequal illumination of the field; rough background subtraction; removal of signals surrounding the chromosome (see Fig. 3 b); determination of lowest and highest gray value in the chromosome by a linear scan (Fig. 3c); base level setting to the lowest gray value; and expansion of the scale of gray levels from 0 to 256 (Fig. $3 \mathrm{~d}$, e). The I-18C region was then boxed-in by use of a mouse and its area and average gray value were determined (see Fig. 3 d) to yield fluorescence value " $a$ ". The same was done for the right arm of chromosome I (Fig. 3d) to yield fluorescence value " $b$ ". The relative fluorescence of the I-18C region was obtained by dividing " $a$ " by " $b$ " (Fig. 4).

\section{Results}

\section{The IEH signal}

Figure $5 \mathrm{~b}$ shows that during oligopause and postoligopause the IEH signal in I-18C undergoes dramatic changes. It is very low in the first half of stage 5 and exhibits two additional minima in stages 6 and 7. This $F_{r}$ pattern is rather puzzling. We therefore undertook several control experiments in order to make sure that it is not due to an artifact.

The reliability of the visual scoring method was checked by comparison with computer-aided image analysis of identical chromosomes in two independent and unbiased runs of evaluations. Figure 4 shows the correlation between data points obtained by these two evaluation methods. It is rather good with a correlation coefficient of 0.971 and an intersection with the $\mathrm{x}$ axis close to zero $(-0.41)$. For calculation of the regression line two groups of points were omitted: (a) points which (for inexplicable reasons) received a wrong score and which in a second (unbiased) round of visual evaluation fell into a distinctly different signal class (i.e., close to the regression line); (b) points from very low I-18C signals. This latter group of points exhibited an $\mathrm{a} / \mathrm{b}$ ratio which we considered too high for the following reasons. There were two steps in the computer-aided procedure which were arbitrary, i.e., definition of the minimal gray value and assessment of the size of the box encompassing the $\mathrm{I}-18 \mathrm{C}$ region. With chromosomes of decreasing activity in I-18C both parameters should approaching zero which was difficult to realize in practice. For the present studies visual scoring was chosen as a routine method because it is faster, less restricted in terms of chromosome I arrangement, and as reliable as computer-assisted image analysis, particularly if applied twice to the same material. Figure 5 a shows the outcome of two such consecutive evaluations.

The stages with low IEH signals in I-18C during oligopause and postoligopause development are also stages with maximal chromosomal condensation of that region. In order to exclude the possibility of steric hindrance of nascent RNA:DNA hybrid formation or of the antibody reaction due to compact chromatin conformation, chromosome preparations were digested with pronase $(1 \mu \mathrm{g} / \mathrm{ml}$ in PBS, $20 \mathrm{~min}$, room temperature) either before or after the denaturation/renaturation step. Although protein removal resulted in swelling of the chromosomes, the $F_{r}$ of I-18C in oligopause stage 5 chromosomes was not changed by such treatment (results not shown). It remained low indicating that the lack of IEH signal was not due to chromatin compaction. However, RNase A plus RNase $\mathrm{H}$ treatment of the preparation abolished all chromosome IEH signals as described previously (Hertner et al. 1986).

If the IEH curve in Figure $5 \mathrm{~b}$ (dashed line) really reflects changes in transcriptional activity of the $\mathrm{I}-18 \mathrm{C}$ region it should be reproduced by other methods revealing gene activity. The anti-RNP antiserum does not require endogenous hybrid formation and has been shown to depict changes in Balbiani ring 1 and 2 activity (unpublished observation) with a course similar to that described previously by other methods (Meyer et al. 1983). Figure 5a shows that the $F_{r}$ values obtained by use of this antiserum follow essentially the same developmental course as those obtained by the IEH method, with minima in stages 5,6 and 7 .

Pulse labeling of explanted glands with $\left[{ }^{3} \mathrm{H}\right]$ uridine measures transcription kinetics rather than the steady state (like 


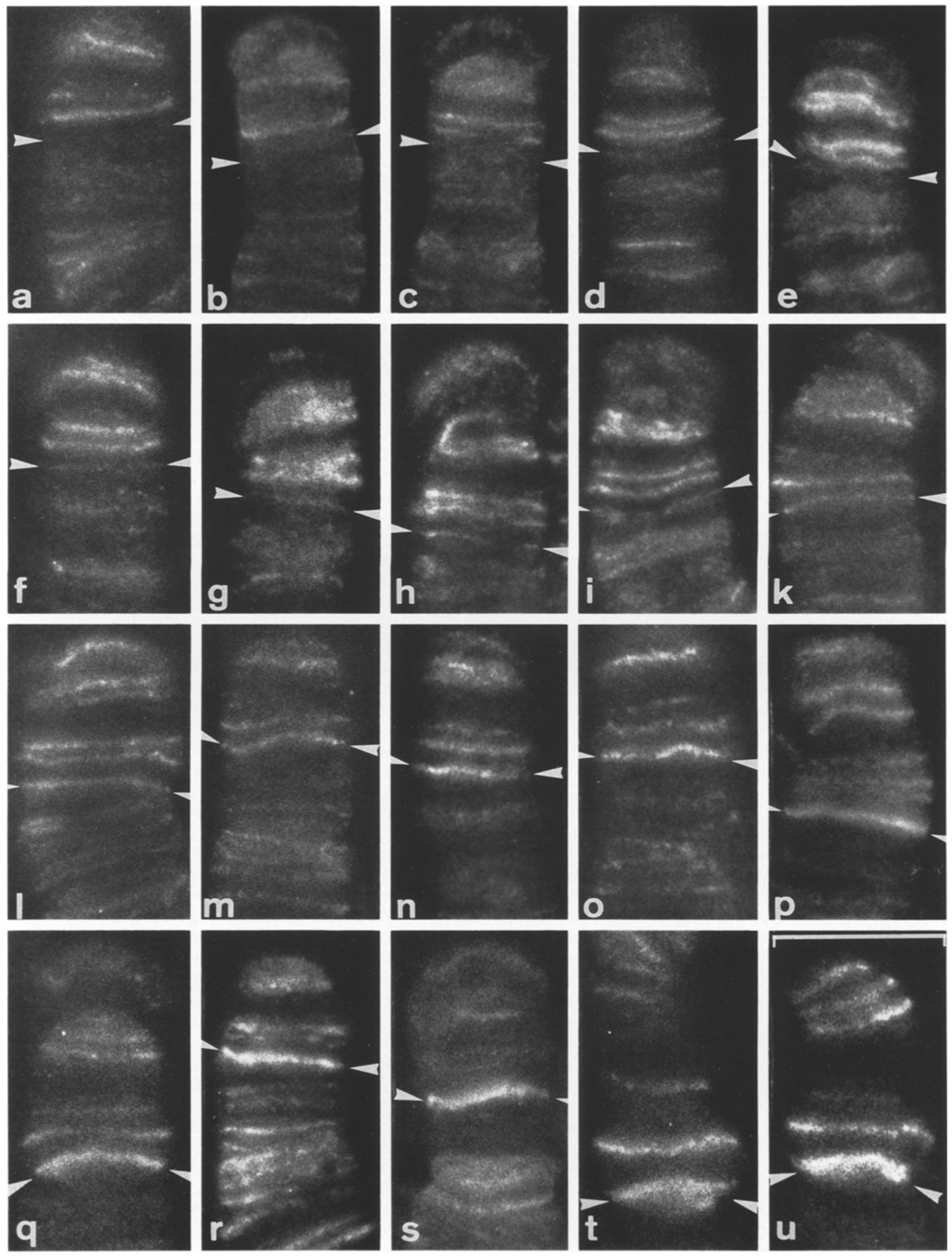

Fig. 2 a-u. Signal classes $(0-19)$ of induced endogenous hybrids (IEH) in region I-18C (arrowheads) as revealed by indirect immunofluorescence. Pictures were selected to show the most continuous increase in the IEH signal of I-18C but otherwise to appear as similar as possible to each other in general IEH pattern and intensity, background and morphology of chromosome I. For that reason pictures of various stages and treatments were compiled, in some instances originating from investigations not included in the present work. Bar represents $10 \mu \mathrm{m}$ 

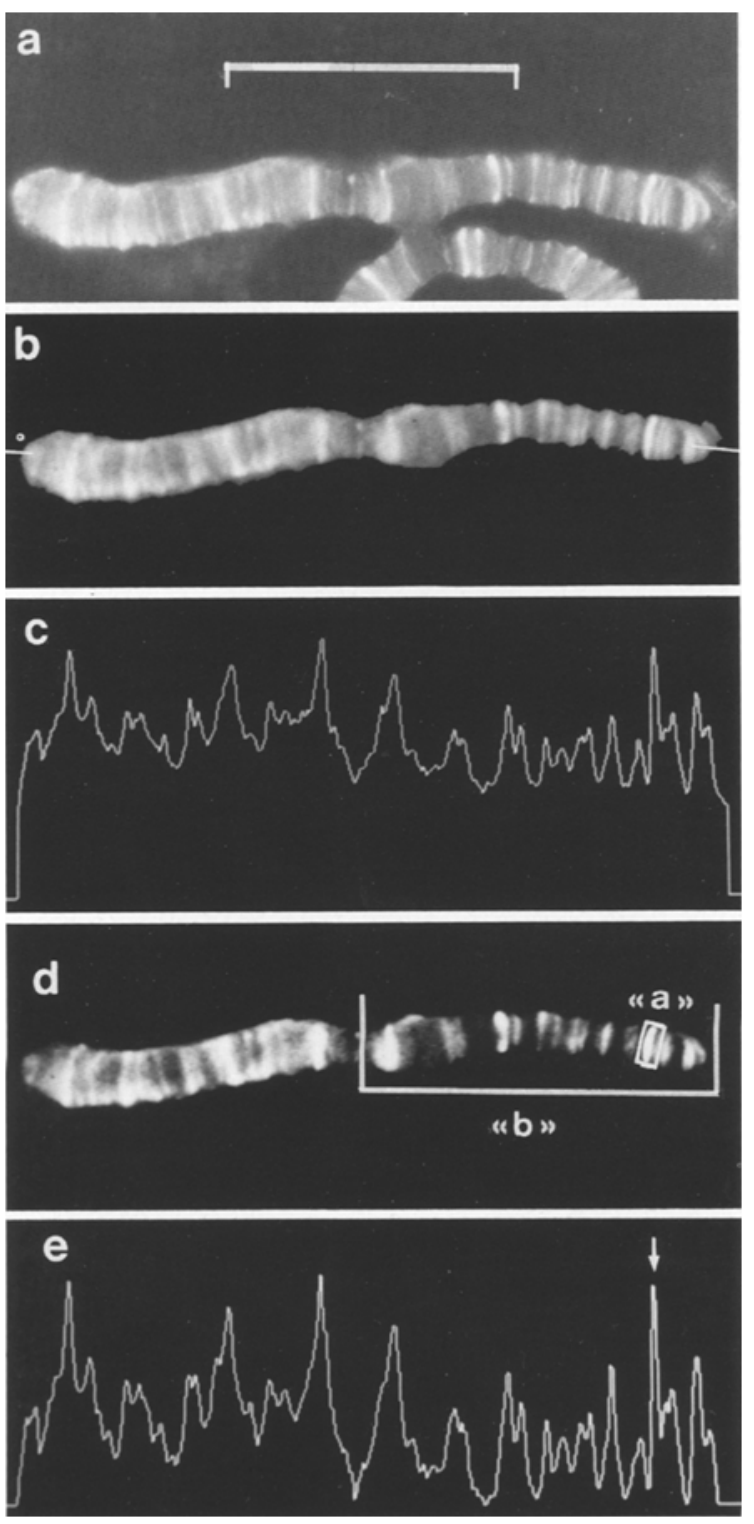

Fig. 3a-e. Computer-assisted image analysis of IEH signal in region I-18C. a Image of chromosome I before processing. b After rough background subtraction and removal of signals outside of chromosome I. c Scanning profile of image shown in $\mathbf{b}$ (beginning and end of scanning line indicated in b). d Final image after subtraction of base level fluorescence (according to scanning profile in c) and subsequent expansion of the scale of gray values between 0 and 256. " $a$ " boxed-in I-18C signal; " $b$ " portion of chromosome I which was boxed-in and used as a reference in calculating the relative IEH signal of I-18C. e Scanning profile of image shown in $\mathbf{d}$ illustrating the limits of linear resolution of the method and the problem of base line setting. Arrow indicates signal in I-18C. Bar represents $50 \mu \mathrm{m}$

the above nascent RNA determinations). Autoradiography is a physical rather than an immunochemical reaction, and is quantitated by grain counting rather than visual scoring. It however, requires (short) in vitro cultivation of the excised tissue in an artificial medium. It is interesting that even this method revealed the activity minima in stages 5 , 6 and 7 (see Fig. 5c). We attribute the quantitative deviations between the two experimental approaches (cf. Fig. 5b and c) not only to the in vitro cultivation step but also to the choice of reference regions used for computing the

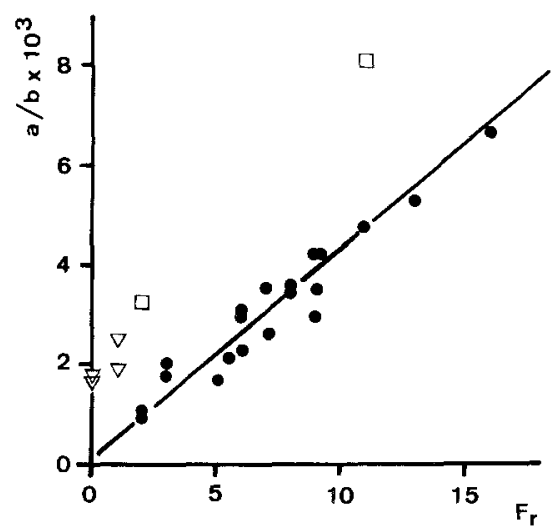

Fig. 4. Evaluation of IEH signal in I-18C; comparison between visual scoring (abscissa) and computer-assisted image analysis (ordinate). Each point represents one chromosome, first evaluated by computer-assisted analysis, second by visual scoring. $\mathrm{F}_{\mathrm{r}}$, relative value of IEH signal in $\mathrm{I}-18 \mathrm{C}$; numbers correspond to classes shown in Figure 2. For determination of $\mathrm{a} / \mathrm{b} \times 10^{3}$, see Figure 3 and text. Filled circles points used for calculation of regression line. Open squares points given a false score by eye. Open triangles points with an IEH value below the sensitivity limits of computer-assisted image analysis (see text)

$G_{r}$ and $F_{r}$ values, respectively. The reference for computing $\mathrm{F}_{\mathrm{r}}$ comprised larger portions of the genome than that for computing $\mathrm{G}_{\mathrm{r}}$.

\section{In vivo application of ecdysterone}

The outcome of the following preliminary studies documents very impressively the difference between oligopause/ postoligopause and subitaneous development in the hormonal effect on chromosome region I-18C (see Table 1). Oligopausing and postoligopausing animals exhibited no significant stimulation of $\mathrm{I}-18 \mathrm{C}$ activity by ecdysterone injection while subitaneously developing animals at stage 5 showed a great stimulation and those at stage 7 at least a significant stimulation. A comparison of the $F_{r}$ value of controls (i.e., of those larvae injected with saline only) with that of non-injected larvae (see Figs. $5 \mathrm{~b}$ and $6 \mathrm{a}$ ) reveals that the injection procedure as such caused an increase in I-18C activity. This effect of larval disturbance was studied in more detail and will be published in a later paper of this series (M. Lezzi et al., in preparation). Animals which were not completely buried in the substrate after $24 \mathrm{~h}$ were omitted from Table 1. These animals (3 out of 10) were possibly wounded by the injection. Their $F_{r}$ values were low whether or not the larvae had received ecdysterone. It is concluded that in vivo ecdysterone application by injection has severe drawbacks notwithstanding the problem of an uncontrollable endocrine situation encountered with intact animal systems. Therefore, in the following experimental series hormonal responsiveness was investigated by an in vitro assay.

\section{Hormonal responsiveness during oligopause and postoligopause development (in vitro studies)}

The hormonal responsiveness of region $\mathrm{I}-18 \mathrm{C}$ is understood as the difference in the $\mathrm{F}_{\mathrm{r}}$ value of its IEH signals observed before and after ecdysterone treatment of the salivary glands in vitro. The incubation of glands was optimized 

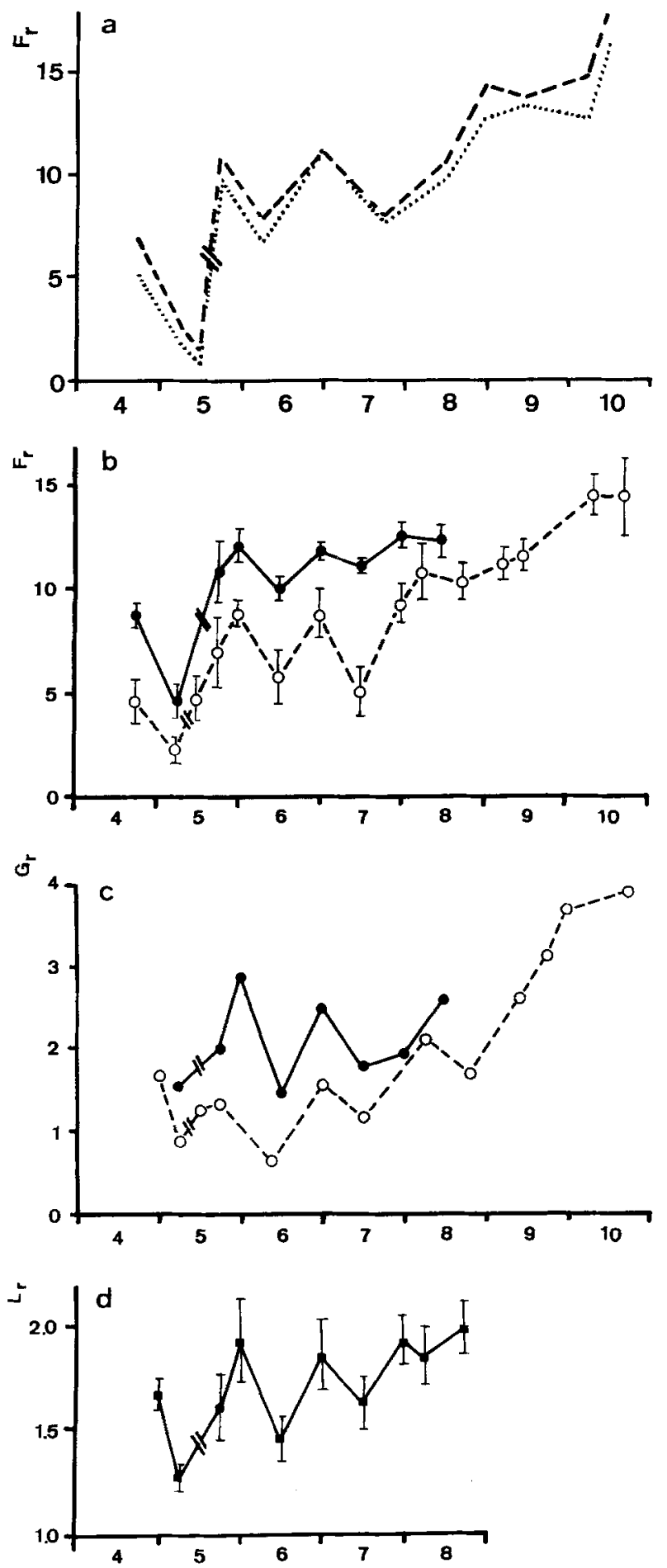

Fig. 5a-d. Behavior of chromosome region I-18C during oligopause and postoligopause development. Stages to the left of interruption in curves represent oligopause; stages to the right of interruption, postoliogopause (stages according to Ineichen et al. 1983). a Relative fluorescent signal $\left(\mathrm{F}_{\mathrm{r}}\right)$ in $\mathrm{I}-18 \mathrm{C}$ produced by indirect immunohistochemistry using anti-RNP antiserum. Dotted and dashed lines represent two independent evaluations of the same material. Basal (i.e., non-induced) state of I-18C. $\mathbf{b} \mathrm{F}_{\mathrm{r}}$ values produced by the IEH method. Dashed line basal activity. Solid line activity after $1 \mathrm{~h}$ in vitro incubation of salivary glands with ecdysterone. c Relative grain number $\left(\mathrm{G}_{\mathrm{r}}\right)$ over $\mathrm{I}-18 \mathrm{C}$ determined in autoradiograms of salivary glands pulse labeled in vitro with $\left[{ }^{3}\right.$ H]uridine. Dashed line pulse labeling in the absence of ecdysterone (revealing basal activity). Solid line pulse labeling at the end to yield a measurable and reproducible effect on I-18C activation within the shortest time possible. One hour incubation at $18^{\circ} \mathrm{C}$ was found to be optimal. Within one hour of in vitro cultivation region $\mathrm{I}-18 \mathrm{C}$ does not become activated spontaneously (i.e., in the absence of ecdysterone), a low extent of wounding might be tolerated, and the responsiveness toward ecdysterone is not changed too much, particularly if the hormone is present right from the beginning of the in vitro incubation. The influence of all these parameters will be dealt with in a subsequent report (M. Lezzi et al., in preparation).

Figure $5 \mathrm{~b}$ shows that the activity (i.e., the IEH signal) of $\mathrm{I}-18 \mathrm{C}$ attained after in vitro ecdysterone treatment changes during oligopause and postoligopause development more or less in parallel with the basal activity of the region. Thus the responsiveness of $\mathrm{I}-18 \mathrm{C}$ in stages 4 to $7 / 8$ remains fairly constant and is rather low in any event, but particularly in stage 5 (see also Fig. $7 \mathrm{a}, \mathrm{b}$ ). Toward the prepupal phase (stage 8 onward) it becomes zero (not all points shown, but see also Lezzi 1974) probably because of the increasing endogenous ecdysterone titer and the limitations of the reaction system. Autoradiographic studies confirm the above findings (Fig. $5 \mathrm{c}$ ). Due to the incomplete sequence of stages the parallelism between the basal and induced curve appears somewhat distorted. The seemingly large responsiveness of stages 6 and 7 observed in the IEH investigations (Fig. 5b) could not be reproduced by the autoradiographic approach (Fig. $5 \mathrm{c}$ ), and vice versa for that of stage $5 / 6$ in the autoradiographic studies.

During oligopause and postoligopause development the induced and basal activity and the state of condensation of chromosome region $\mathrm{I}-18 \mathrm{C}$ show cyclical changes (see Fig. 5d). The region is most condensed in the first half of stage 5 in oligopause, decondenses after breakage of oligopause, and recondenses in stage 6 of postoligopause with a repetition of that cycle in stages 7 and maybe 8 .

The conclusions to be drawn from the findings on I-18C during oligopause and postoligopause seem to be rather simple. After breakage of oligopause I-18C forms a puff which regresses somewhat and reforms twice until it develops fully with the onset of metamorphosis. The "puff" may be perceived (within the limits of the above studies) in the conventional way as a morphological manifestation of locally increased transcription. The effect of ecdysterone, then, would simply be a modulation of the preexisting puff situation, i.e., a modest enhancement of $\mathrm{I}-18 \mathrm{C}$ activity.

\section{Hormonal responsiveness during subitaneous development (in vitro studies)}

A quick glance at Figure 6 a discloses that the above conclusions cannot be generally true. The developmental course of induced activity in subitaneous larvae does not just follow that of basal activity. During the period from the beginning of stage 5 to the end of stage 6 induced activity is

of $1 \mathrm{~h}$ incubation in the presence of ecdysterone, $\mathbf{d}$ Decondensation of $\mathrm{I}-18 \mathrm{C}$ region as determined by its relative length $\left(\mathrm{L}_{\mathrm{r}}\right)$. The glands were not incubated (thus, non-induced) but fixed immediately after decapitation of larvae. Vertical bars in $\mathbf{b}$ and $\mathbf{d}$ indicate $95 \%$ confidence limits. Total number of chromosomes evaluated: a 139, b 738 , c 586, d 319 
Table 1. Influence of stage and mode of development on response of chromosome region I-18C to in vivo application of ecdysterone

\begin{tabular}{lllll}
\hline Mode of development & Stage & $\begin{array}{l}\text { Substance injected } \\
(1 \mu \mathrm{l})\end{array}$ & $\begin{array}{l}\text { Number of } \\
\text { chromosomes evaluated }\end{array}$ & $\begin{array}{l}\left.\text { Fluorescent signal (F } \mathrm{F}_{\mathrm{r}}\right) \\
\text { of IEH in I-18C, } \\
24 \mathrm{~h} \text { after injection } \\
( \pm 95 \% \text { confidence limits) }\end{array}$ \\
\hline Oligopause & 5 & Control $^{\mathrm{a}}$ & 24 & $9.7 \pm 0.87$ \\
Oligopause & 5 & Ecdysterone & 23 & $11.3 \pm 1.27$ \\
Postoligopause & 7 & Control & 21 & $9.5 \pm 0.68$ \\
Postoligopause & 7 & Ecdysterone & 11 & $10.4 \pm 0.45$ \\
Subitaneous & 5 & Control & 23 & $7.1 \pm 1.0$ \\
Subitaneous & 5 & Ecdysterone & 23 & $14.7 \pm 0.7$ \\
Subitaneous & 7 & Control & 20 & $8.9 \pm 0.9$ \\
Subitaneous & 7 & Ecdysterone & 21 & $13.4 \pm 0.6$ \\
\hline
\end{tabular}

a $0.65 \% \mathrm{NaCl}$

b $1.6 \times 10^{-5} \mathrm{M}$ ecdysterone in $0.65 \% \mathrm{NaCl}$
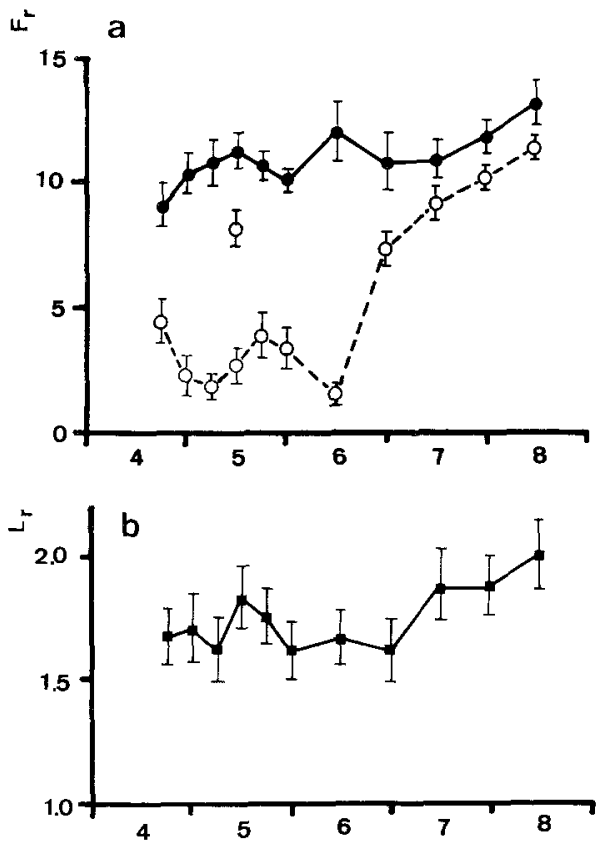

Fig. 6a, b. Behavior of chromosome region I-18C during subitaneous development. a IEH method revealing basal activity (dashed line no incubation) and induced activity (solid line) obtained by incubating salivary glands with ecdysterone for $1 \mathrm{~h}$. b I-18C decondensation (no incubation of salivary glands). Vertical bars indicate $95 \%$ confidence limits. Total number of chromosomes evaluated: a 572 , b 219

generally high while the basal activity is low (see also Fig. $7 \mathrm{c}$, d) resulting in a normally high responsiveness in these stages. Even some smaller variations in each curve do not parallel each other but rather behave reciprocally. It is interesting to note, that the basal activity curve during subitaneous development is reminiscent of that observed during oligopause and postoligopause development with depressions in stages 5 (first half) and 6 . The peak in the second half of stage 5 suggests that a fraction of the subitaneous larval population is ready to follow momentarily the developmental pattern of larvae breaking through their resting state.

In contrast to the basal activity the course of I-18C decondensation is paralleled rather nicely by that of induced activity (Fig. 6b). It is generally high and never reaches
$\mathrm{L}_{\mathrm{r}}$ values as low as those attained during oligopause. One would expect to find a small puff in I-18C in untreated subitaneous larvae of stages 5 to 6 . However, puffing in the true sense does not occur (see Fig. 7c, f). Real puffing was not observed earlier than stage 7 , i.e., when transcription in I-18C commences. Prior to that stage, the I-18C region of subitaneous larvae just looks less tight and wider (in the longitudinal axis) than it does in oligopausing larvae (Fig. 7f). Chromosomes from subitaneous development differ from oligopause chromosomes in their general appearance; they look lighter and more slender than the latter (cf. Fig. $7 \mathrm{f}$ and e). However, it has to be emphasized that decondensation in region $\mathrm{I}-18 \mathrm{C}$ of subitaneous chromosomes is nevertheless exceptionally pronounced (N.B. its $L_{r}$ value is obtained by standardization with the length of region $\mathrm{I}-18 \mathrm{~B}$ ) and that the higher degree of compaction of oligopause chromosomes (Fig. 7e) does not depend on a higher level of polyteny.

It may, thus, be concluded that a specific state of decondensation of chromosome region $\mathrm{I}-18 \mathrm{C}$ exists which differs from puffing in that it does not reflect locally increased transcription. However, it does seem to be related to the hormonal responsiveness of the region.

\section{Discussion}

\section{Significance of the IEH signal}

We showed that the course of activity in I-18C during oligopause and postoligopause development is more or less the same whether it is determined by the IEH method, by use of anti-RNP antiserum or autoradiographically after $\left[{ }^{3} \mathrm{H}\right]$ uridine incorporation. However, some questions concerning the quantitative results obtained by the IEH method remain to be discussed. Since one full-length nascent RNA molecule will cover up (i.e., by hybrid formation) the whole transcription unit of the transcribed DNA single strand it is obvious that any additional nascent RNA molecule being simultaneously transcribed from that same strand cannot also form a hybrid. Thus, endogenous hybrid formation is not linearly correlated with the number of nascent RNAs in very actively transcribed genes and is therefore less suitable for studying such genes quantitatively. Balbiani ring genes and ribosomal genes are known to be hyperactive and to exhibit staggering of many nascent RNA molecules at the same strand. However, these genes 

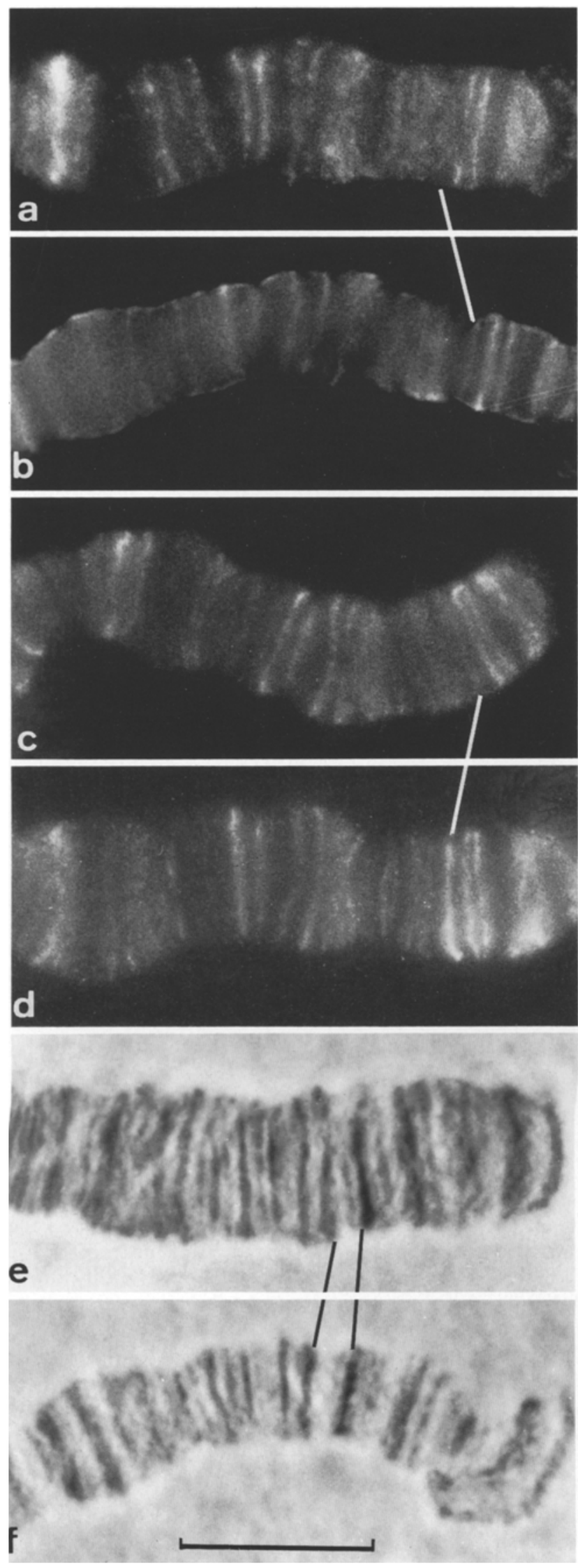

seem to be exceptional. Normal genes (like those in regular puffs) appear to be involved in transcription of many fewer RNA molecules at a time (cf. Laird and Chooi 1976). Moreover, we were interested in events occurring at the onset of activation of region $\mathrm{I}-18 \mathrm{C}$. For such a situation the IEH method is ideal. It is very sensitive because one hybrid molecule may be recognized by several antibody molecules (cf. Malfoy et al. 1982) and its resolution is high. It is superior to that of autoradiography but also to that of the anti-RNP immunoreaction which produces less distinct signals. The IEH signal is a measure of how many base pairs of DNA are hybridized by RNA. Kinetic considerations led Alcover et al. (1982) to conclude that this RNA must be very close to the DNA in order to undergo hybrid formation within the given time, i.e., it is RNA attached to DNA. Diez and Barettino (1984) assumed that this RNA represents not only transcripts in statu nascendi but also finished transcripts not yet released from their template. In the context of our experiments the problem of stored RNA is not too serious because we studied the activation response to ecdysterone rather than the process of inactivation of previously active loci (which may retain their RNA). The resolution of the IEH method by use of anti-hybrid antibodies is high and allowed us to study different subregions of the $\mathrm{I}-18 \mathrm{C}$ region separately. Under the conditions used in the present work we discerned an IEH signal at one specific place only, i.e., close to the I-18C/19A boundary where Pelling (1964) had mapped the only puff he could observe in the whole I-18C region. Despite the good resolution it must be realized that the DNA subregion spanned by the IEH signal could still encompass more than one gene. Thus, it is impossible to decide whether or not the developmental changes we observed in I-18C result from changes in activity of one or more than one gene.

We have cloned a gene from I-18C (Hertner et al. 1986). Using an indirect hybridization method for revealing nascent transcripts of that cloned gene clear differences between oligopause (stage 5) and prepupae could be found, as well as between control and ecdysterone-treated salivary glands (Hertner et al. 1986). The cloned gene is therefore a good candidate for a gene producing the IEH signal studied in the present investigations. It remains to be elucidated whether or not it is the only gene contributing to the IEH signal in $\mathrm{I}-18 \mathrm{C}$.

\section{Comparison between oligopause/postoligopause and subitaneous development}

Oligopause is a special form of diapause. In $C$. tentans as in $C$. plumosus and $C$. nuditarsis it occurs in the middle of the fourth instar, and it is induced and terminated largely

Fig. 7 a-f. Comparison of basal activity (a, c), ecdysterone-induced activity $(\mathbf{b}, \mathbf{d})$, and state of chromatin decondensation $(e, f)$ of chromosome region I-18C in oligopause $(\mathbf{a}, \mathbf{b}, \mathbf{e})$ and subitaneously developing (c, d, f) stage 5 larvae. a-d IEH signals revealed by indirect immunofluorescence; white lines point to IEH signal in I-18C. e-f Phase contrast photographs (green filter) of orcein/fast green-stained chromosomes of non-induced (untreated) salivary glands. Black lines indicate the $\mathrm{I}-18 \mathrm{C}$ region. Note the greater induction of I-18C activity by ecdysterone in a subitaneous gland (d) and the lighter and wider appearance of the $\mathrm{I}-18 \mathrm{C}$ region in an untreated gland of subitaneous larva (f). Bar represents $10 \mu \mathrm{m}$ 
by changes in the diurnal light regimen controlling hibernation (Fischer 1974). The exact mechanism of oligopause induction and termination in Chironomids has not yet been unraveled. It is likely that hormones (e.g., ecdysterone) are involved. Valentin et al. (1978) have determined the ecdysterone titer in hemolymph of resting (oligopausing) $C$. thummi larvae to be low, suggesting that the absence of this hormone might be crucial for the maintenance of oligopause. The fate of the ecdysterone titer during and after breakage of oligopause has not yet been investigated. Assuming that transcriptional activity in $\mathrm{I}-18 \mathrm{C}$ is coupled to ecdysterone action the activity burst in stage $5 / 6$ of postoligopausing larvae could be explained by a sudden rise in the endogenous ecdysterone titer. It would then fall back, recover and fall back again until, with the onset of the prepupal period (about stage 8), it would start rising definitively. In keeping with the same assumption it would be predicted that during subitaneous development the premature upsurge of the ecdysterone titer in stage $5 / 6$ is normally suppressed and that the rise toward pupation is more continuous. However, the results of experiments in which the ecdysterone titer is increased artificially (i.e., in tissue culture medium) reveal that the above interpretation is too simplistic. With oligopause stage 5 glands the depression in basal activity of I-18C cannot be overcome simply by supplying high enough ecdysterone doses; this is quite in contrast to subitaneous stage 5 glands. It must be concluded that in oligopause stage 5 the system is rendered refractory toward the hormone. If so, one cannot even be sure that a low basal activity necessarily indicates a low endogeneous ecdysterone titer. The conclusion is that during stage 5 of oligopause a superimposed control mechanism must be functioning which is not directly dependent on the ecdysterone titer.

The relation between hormonal responsiveness, basal activity, and local chromatin decondensation of region I-18C

Changes in steroid responsiveness of target tissues have been described previously (for review, see Milgrom et al. 1973). Refractory periods were explained in terms of steroid receptor depletion in the target cell (Milgrom et al. 1973) or of an autoinhibitory action of a hormone-induced gene product (negative feedback, see e.g., Ashburner et al. 1974). In principle, both models could be applied to the relatively high refractoriness of oligopausing larvae in stage 5. Undoubtedly stage 5 salivary glands of fourth instar larvae have experienced an ecdysterone stimulation previously, that is, during the last molting cycle. Thus, hormone dependent receptor translocation and inactivation or hormoneinduced "repressor" production may have occurred. However, subitaneously developing larvae of stage 5 are much closer to such an event (days versus months) than oligopausing animals. Nevertheless, they are not refractory to ecdysterone in any stage tested between 4.5 and 8 . Furthermore, extensive studies by Clever (1966) have demonstrated that puff I-18C undergoes no induction/repression cycle in the continuous presence of ecdysterone, in complete contrast to puff IV-2B of C. tentans and the early ecdysteroneinducible puffs of Drosophila (Ashburner et al. 1974). We believe that the hormone refractoriness of region $\mathrm{I}-18 \mathrm{C}$ in oligopause is brought about by a mechanism different from those cited above. Observations to be published later will substantiate this point.
We are intrigued by the parallelism between the extent of hormone-inducible activity and the preexisting degree of decondensation of chromosome region I-18C, and are inclined to see a causal relationship between the two parameters. This is in contrast to the course of basal activity which sometimes does and sometimes does not parallel that of induced activity (cf. oligopause/postoligopause versus subitaneous development). With subitaneous larvae local decondensation of $\mathrm{I}-18 \mathrm{C}$ results in a mere loosening of the chromatin structure while with postoligopausing animals it results in a true puff, i.e., it is accompanied by transcriptional activity. This functional rather than structural difference is possibly due to differences in the endogeneous ecdysterone titer between the two groups of animals (see above). Chromatin compaction and hormonal responsiveness could be connected to each other in a way outlined by the multistep model published recently (Lezzi and Richards 1989). According to this model the state of chromatin condensation determines the accessibility of the hormone receptor to regulatory DNA elements of ecdysterone-activated genes. The question of how, in turn, the state of chromatin is regulated remains largely obscure, although preliminary observations indicate an involvement of intracellular ion activity changes (M. Lezzi et al., manuscript in preparation).

Acknowledgements. This work was supported by a grant of the Swiss National Science Foundation to M.L. We thank Drs. B. Dworniczak and $\mathrm{O}$. Pongs for providing us with the anti-ecdysteroid antiserum and Dr. B.D. Stollar for antibodies against RNA:DNA hybrids. We are grateful to Dr. T.M. Jovin for use of his facilities.

\section{References}

Alcover A, Izquierdo M, Stollar BD, Kitagawa Y, Miranda M, Alonso C (1982) In situ immunofluorescent visualization of chromosomai transcripts in polytene chromosomes. Chromosoma $87: 263-277$

Arndt-Jovin DJ, Robert-Nicoud M, Baurschmidt P, Jovin TM (1985) Immunofluorescence localization of Z-DNA in chromosomes: Quantitation by scanning microphotometry and computer-assisted image analysis. J Cell Biol 101:1422-1433

Ashburner M, Chihara C, Meltzer P, Richards G (1974) Temporal control of puffing activity in polytene chromosomes. Cold Spring Harbor Symp Quant Biol 38:655-662

Beermann W (1955) Geschlechtsbestimmung und Evolution der genetischen Y-Chromosomen bei Chironomus. Biol Zentralb1 74:525-544

Büsen W, Amabis M, Leoncini O, Stollar BD, Lara FJS (1982) Immunofluorescent characterization of DNA-RNA hybrids on polytene chromosomes of Trichosia pubescens (Diptera, Sciaridae). Chromosoma 87:263-277

Clever U (1966) Induction and repression of a puff in Chironomus tentans. Dev Biol 14:421-438

Clever U, Karlson P (1960) Induktion von Puff-Veränderungen in den Speicheldrüsenchromosomen von Chironomus tentans durch Ecdyson. Exp Cell Res 20:623-626

Clever U, Clever I, Storbeck I, Young NL (1973) The apparent requirement of two hormones, $\alpha$ - and $\beta$-ecdysone, for molting induction in insects. Dev Biol 31:47-60

Diez JL, Barettino D (1984) DNA-RNA hybrids and transcriptional activity in Chironomus polytene chromosomes. Chromosoma $90: 103-110$

Dworniczak B, Seidel R, Pongs O (1983) Puffing activities and binding of ecdysteroid to polytene chromosomes of Drosophila melanogaster. EMBO J 2:1323-1330

Fischer J (1974) Experimentelle Beiträge zur Oekologie von Chi- 
ronomus (Diptera). I. Dormanz bei Chironomus nuditarsis und Ch. plumosus. Oecologia 16:73-95

Hertner T, Meyer B, Eppenberger HM, Mähr R (1980) The secretion proteins in Chironomus tentans salivary glands: Electrophoretic characterization and molecular weight estimation. Wilhelm Roux's Arch 189:69-72

Hertner T, Hertner-Meyer B, Sogo JM, Schläpfer G, Jäckle H, Edström JE, Lezzi M (1986) Microcloning of a long segment of a gene from the primary ecdysterone-controlled puff region I-18C of Chironomus tentans: Molecular characterization and effect of hormone on its transcription. Dev Biol 113:29-39

Ineichen H, Meyer B, Lezzi M (1983) Determination of the developmental stage of living fourth instar larvae of Chironomus tentans. Dev Biol 98:278-286

Kitagawa Y, Stollar BD (1982) Comparison of poly(A)-poly(dT) and poly $(\mathrm{I})$-poly $(\mathrm{dC})$ as immunogens for the induction of antibodies to RNA-DNA hybrids. Mol Immunol 19:413-420

Laird CD, Chooi WY (1976) Morphology of transcription units in Drosophila melanogaster. Chromosoma 58:193-218

Langer-Safer PR, Levine M, Ward DC (1982) Immunological method for mapping genes on Drosophila polytene chromosomes. Proc Natl Acad Sci USA 79:4381--4385

Lezzi M (1974) In vitro effects of juvenile hormone on puffing in Chironomus salivary glands. Mol Cell Endocrinol 1:189-207

Lezzi M (1984) Heat-shock phenomena in Chironomus tentans. II. In vitro effects of heat and overheat on puffing and their reversal. Chromosoma 90:198-203

Lezzi M, Kroeger H (1966) Aufnahme von ${ }^{22} \mathrm{Na}$ in die Zellkerne der Speicheldrüse von Chironomus thummi. Z Naturforsch $21: 274-277$

Lezzi M, Richards G (1989) Models for the study of ecdysteroid action. Salivary glands. In: Koolman J (ed) Ecdysone. Eine Festschrift. Thieme Verlag, Stuttgart (in press)

Lezzi M, Gatzka F, Meyer B (1984) Heat-shock phenomena in Chironomus tentans. III. Quantitative autoradiographic studies on ${ }^{3} \mathrm{H}$-uridine incorporation into Balbiani ring 2 and heatshock puff IV-5C. Chromosoma 90:204-210

Lezzi M, Meyer B, Mähr R (1981) Heat shock phenomena in Chironomus tentans. I. In vivo effects of heat, overheat, and quenching on salivary chromosome puffing. Chromosoma 83 : 327-339

Malfoy B, Rousseau N, Leng M (1982) Interaction between antibodies to Z-form deoxyribonucleic acid and double-stranded polynucleotides. Biochemistry $21: 5463-5467$

Meyer B, Mähr R, Eppenberger HM, Lezzi M (1983) The activity of Balbiani rings 1 and 2 in salivary glands of Chironomus tentans larvae under different modes of development and after pilocarpine treatment. Dev Biol 98:265-277

Milgrom E, Luu Thi M, Baulieu EE (1973) Control mechanisms of steroid hormone receptors in the reproductive tract. In: Karolinska Symposia on Research Methods in Reproductive Endocrinology, pp 380-403

Pelling C (1964) Ribonukleinsäure-Synthese der Riesenchromosomen. Autoradiographische Untersuchungen an Chironomus tentans. Chromosoma 15:71-122

Valentin M, Bollenbacher WE, Gilbert LI, Kroeger H (1978) Alterations in ecdysone content during the post-embryonic development of Chironomus thummi: Correlations with chromosomal puffing. Z Naturforsch 33e:557-560

Widmer RM, Lucchini R, Lezzi M, Meyer B, Sogo JM, Edström JE, Koller T (1984) Chromatin structure of a hyperactive secretory protein gene (in Balbiani ring 2) of Chironomus. EMBO J $3: 1635-1641$

Wyss C (1982) Chironomus tentans epithelial cell lines sensitive to ecdysteroids, juvenile hormone, insulin and heat shock. Bxp Cell Res 139:309-319

Received

Accepted by W. Beermann 